\title{
Thrombin-induced miRNA-24-1-5p upregulation promotes angiogenesis by targeting prolyl hydroxylase domain 1 in intracerebral hemorrhagic rats
}

\author{
Hanjin Cui, MD, PhD, ${ }^{1}$ Ali Yang, MD, PhD, ${ }^{2}$ Huajun Zhou, MD, PhD, ${ }^{3}$ Yang Wang, MD, PhD, ${ }^{1}$ \\ Jiekun Luo, MD, PhD, ${ }^{1}$ Jun Zhou, MD, ${ }^{4}$ Tao Liu, MD, PhD, ${ }^{1}$ Pengfei Li, MD, PhD, ${ }^{1}$ \\ Jing Zhou, MD, PhD, ${ }^{1}$ En Hu, MD, ${ }^{1}$ Zehui He, MD, ${ }^{1}$ Wang Hu, MD, ${ }^{1}$ and Tao Tang, MD, PhD' \\ ${ }^{1}$ Institute of Integrative Medicine and ${ }^{4}$ Institute of Medical Science, Xiangya Hospital, Central South University, Changsha, \\ Hunan; 'Department of Neurology, Henan Province People's Hospital, Zhengzhou; and ${ }^{3}$ nstitute of Neurology, The First College \\ of Clinical Medical Sciences, China Three Gorges University, Yichang, Hubei, China
}

OBJECTIVE Thrombin is a unique factor that triggers post-intracerebral hemorrhage (ICH) angiogenesis by increasing hypoxia-inducible factor-1a (HIF-1a) at the protein level. However, HIF-1a mRNA remains unchanged. MicroRNAs (miRNAs) mediate posttranscriptional regulation by suppressing protein translation from mRNAs. This study aimed to determine if miRNAs might be involved in thrombin-induced angiogenesis after ICH by targeting HIF-1a or its upstream prolyl hydroxylase domains (PHDs).

METHODS The study was divided into two parts. In part 1, rats received an injection of thrombin into the right globus pallidus. An miRNA array combined with miRNA target prediction, luciferase activity assay, and miRNA mimic/inhibitor transfection were used to identify candidate miRNAs and target genes. Part 2 included experiments 1 and 2. In experiment 1 , rats were randomly divided into the sham group, $\mathrm{ICH}$ group, and $\mathrm{ICH}+$ hirudin-treated (thrombin inhibitor) group. In experiment 2, the rats were randomly divided into the sham group, $\mathrm{ICH}$ group, $\mathrm{ICH}+$ antagomir group, ICH+antagomircontrol group, and $\mathrm{ICH}+$ vehicle group. Western blotting and quantitative real-time polymerase chain reaction were used to determine the expression of protein and miRNA, respectively. The coexpression of miR-24-1-5p (abbreviated to miR24) and von Willebrand factor was detected by in situ hybridization and immunohistochemical analysis. The angiogenesis was evaluated by double-labeling immunofluorescence. Neurological function was evaluated by body weight, modified Neurological Severity Scores, and corner turn and foot-fault tests.

RESULTS In part 1, it was shown that miR-24, which is predicted to target PHD1, was upregulated (fold-change of 1.83) after thrombin infusion, and that the miR-24 mimic transfection decreased luciferase activity and downregulated PHD1 expression ( $p<0.05)$. miR-24 inhibitor transfection increased PHD1 expression $(p<0.05)$. In part 2, it was shown that miR-24 was expressed in endothelial cells. The HIF-1a protein level and proliferating cell nuclear antigen-positive $\left(\mathrm{PCNA}^{+}\right)$nuclei in vessels were increased, while the PHD1 protein level was decreased after $\mathrm{ICH}$, and these effects were reversed by hirudin $(p<0.05)$. The antagomiR-24-treated rats exhibited a markedly lower body weight and significantly poorer recovery from neurological deficit compared with those in ICH groups $(p<0.05)$. AntagomiR-24 intervention also led to lower miR-24 expression, a higher PHD1 protein level, and fewer PCNA ${ }^{+}$nuclei in vessels compared with those in ICH groups $(p<0.05)$.

CONCLUSIONS The present study suggests that thrombin reduces HIF-1a degradation and initiates angiogenesis by increasing miR-24, which targets PHD1 after ICH.

https://thejns.org/doi/abs/10.3171/2020.2.JNS193069

KEYWORDS thrombin; microRNAs; angiogenesis; prolyl hydroxylase domain 1; intracerebral hemorrhage; vascular disorders

ABBREVIATIONS BMEC = brain microvascular endothelial cell; CTT = corner turn test; $\mathrm{EC}=$ endothelial cell; $\mathrm{FFT}=$ foot-fault test; $\mathrm{HIF}=$ hypoxia-inducible factor; ICH = intracerebral hemorrhage; IHC = immunohistochemistry; ISH = in situ hybridization; LCM = laser capture microdissection; miRNA = microRNA; mNSS = modified Neurological Severity Score; PCNA = proliferating cell nuclear antigen; PHD = prolyl hydroxylase domain; $q \mathrm{RT}-\mathrm{PCR}=$ quantitative real-time polymerase chain reaction; $v$ WF = von Willebrand factor.

SUBMITTED November 11, 2019. ACCEPTED February 24, 2020.

INCLUDE WHEN CITING Published online May 15, 2020; DOI: 10.3171/2020.2.JNS193069. 
I NTRACEREBRAL hemorrhage (ICH) accounted for $10 \%-$ $15 \%$ of all strokes worldwide during the last decade. It is characterized by a high mortality rate in its early stage and poor functional outcomes in most cases. ${ }^{1}$ However, until recently, there have been no effective surgical or medical treatments available to improve the prognosis of patients with $\mathrm{ICH} .^{2}$ Thus, understanding the underlying mechanisms and identifying novel therapeutic targets are required for $\mathrm{ICH}$ treatment.

We previously demonstrated the occurrence of angiogenesis following $\mathrm{ICH} .{ }^{3}$ Angiogenesis is a reparative process; it increases the local blood and oxygen supply in damaged regions and speeds up metabolite exchange and toxic substance removal. During this process, numerous relevant cytokines, chemokines, prostaglandins, and proteases are upregulated, ${ }^{4}$ including vascular endothelial growth factor, angiopoietin 1, and hypoxia-inducible factor-1 $\alpha(\mathrm{HIF}-1 \alpha))^{3,5,6} \mathrm{HIF}-1 \alpha$ is the inducible subunit of HIF-1. As the nuclear transcriptional factor of various proangiogenic factors, HIF-1 is the initial point and the central regulator of angiogenesis.

However, unlike in ischemic stroke, the perihemor-

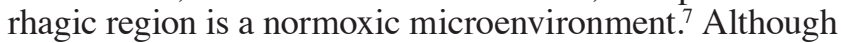
HIF-1 $\alpha$ expression is generally considered to be increased by hypoxia, there is still accumulating evidence showing that under normoxic conditions, many growth factors, cytokines, and other signaling molecules can activate HIF$1 \alpha .8$ Thrombin is a critical signaling molecule cleaved from the proenzyme prothrombin after bleeding. ${ }^{10}$ Our previous study demonstrated that thrombin is a unique factor for initiating post-ICH angiogenesis and increasing $\mathrm{HIF}-1 \alpha$ at the protein level. Intriguingly, HIF- $1 \alpha$ mRNA showed no change, ${ }^{3,11}$ which piqued our interest.

MicroRNAs (miRNAs) are small, noncoding RNA molecules that mediate posttranscriptional regulation by degrading mRNA or suppressing protein translation, during which they bind to complementary sequences of the target mRNAs according to the rule of perfect or imperfect base-pairing. ${ }^{12}$ miRNAs participate in a wide range of biological processes, including angiogenesis. ${ }^{13}$ Therefore, we hypothesized that miRNA might be involved in thrombin-induced angiogenesis after ICH by targeting HIF-1 $\alpha$. However, we failed to find differentially expressed miRNAs directly targeting HIF-1 $\alpha$ using TaqMan Array MicroRNA Cards (Exiqon) combined with miRNA target prediction databases. Interestingly, we identified increased expression of miR-24 (short for miR-24-1-5p) targeting prolyl hydroxylase domain 1 (PHD1) in the current study. Various posttranslational modifications regulate the activity and the stability of HIF-1 $\alpha .{ }^{23}$ Hydroxylation mediated by HIF PHD proteins is one of the most important posttranslational modifications. ${ }^{14}$ Under hypoxia, oxygendependent PHDs are suppressed, and the degradation of HIF-1 $\alpha$ protein by PHDs is decreased as well. ${ }^{15}$ However, hypoxic stress following ICH is mild and transient. ${ }^{3}$ Thus, PHD1-targeting miRNA might be another way to control the level of PHDs through which the HIF-1 $\alpha$ degradation is depressed to promote angiogenesis after $\mathrm{ICH}$.

In the present study, we first screened thrombin-altered miRNAs using an miRNA array (Exiqon). Next, PHD1targeting miR-24 was found by bioinformatics analysis and luciferase activity assay. The interaction between miR-24 and PHD1 was identified by the mimic/inhibitor transfection combined with Western blotting. The role of thrombin in miR-24 alteration following ICH was evaluated by using the thrombin inhibitor hirudin. Finally, the miRNA antagonist antagomiR-24 was used to further validate whether miR-24 can decrease PHD1 expression after $\mathrm{ICH}$. The findings of the present study indicated that thrombin increases HIF- $1 \alpha$ by PHD1-targeting miR-24, thereby inducing angiogenesis after $\mathrm{ICH}$, and thrombinmiR-24/PHD1/HIF-1 $\alpha$ is a reparative pathway involved in post-ICH tissue restoration.

\section{Methods}

\section{Experimental Protocol}

The timeline of the study design is shown in Fig. 1. The present study contained two parts. In part 1, 4 rats received an injection of thrombin (1 U; Sigma-Aldrich) into the right globus pallidus. Seven days after thrombin injection, the globus pallidus was obtained from either hemisphere for the miRNA array to determine differential expression of miRNAs. Differentially expressed miRNAs were predicted using bioinformatics analysis. Luciferase activity assay, mimic/inhibitor transfection, and Western blotting were performed to confirm the interaction between candidate miRNAs and their target genes.

Part 2 included experiments 1 and 2 . In experiment 1 , 135 rats were randomly assigned to 3 groups: the sham control group, ICH group, and $\mathrm{ICH}+$ hirudin-treated group (15 rats for each time point in each group). The randomization of rats was performed as described by Cheng et al. ${ }^{16}$ In this experiment, the rats were injected with $100 \mu \mathrm{l}$ of autologous whole blood with or without hirudin (5 U; Sigma-Aldrich) into the right globus pallidus. The sham controls received $100 \mu \mathrm{l}$ of $0.9 \%$ sterile saline at the same site. In experiment 2, 150 rats were randomly divided into 5 groups: the sham control group, $\mathrm{ICH}$ group, ICH+antagomir group (miRNAs inhibitor), and ICH+antagomir-control (ICH+antagomir-Co) and $\mathrm{ICH}+$ vehicle groups (15 rats for each time point in each group). ICH rats were infused with $0.9 \%$ sterile saline, antagomir, antagomir negative control, and antagomir vehicle, respectively, into the lateral ventricle. In total, 3 rats died from ICH induction. Rats that died before the end point were excluded and replaced with new rats.

\section{Animal Preparation}

Sprague Dawley rats (adult males, 180-220 g at the time of $\mathrm{ICH}$ ) were obtained from the Experimental Animal Science Center of Central South University. The Institutional Animal Care and Use Committee and ethics committee of Central South University approved the protocols for these studies. All parts of the present study adhered to the ARRIVE guidelines. ${ }^{17}$ The details are shown in the Supplemental Data.

\section{Thrombin Treatment and ICH Model}

Thrombin treatment and ICH model establishment was based on our previous study. ${ }^{18,19}$ The details are shown in the Supplemental Data. The detailed methods for tissue 

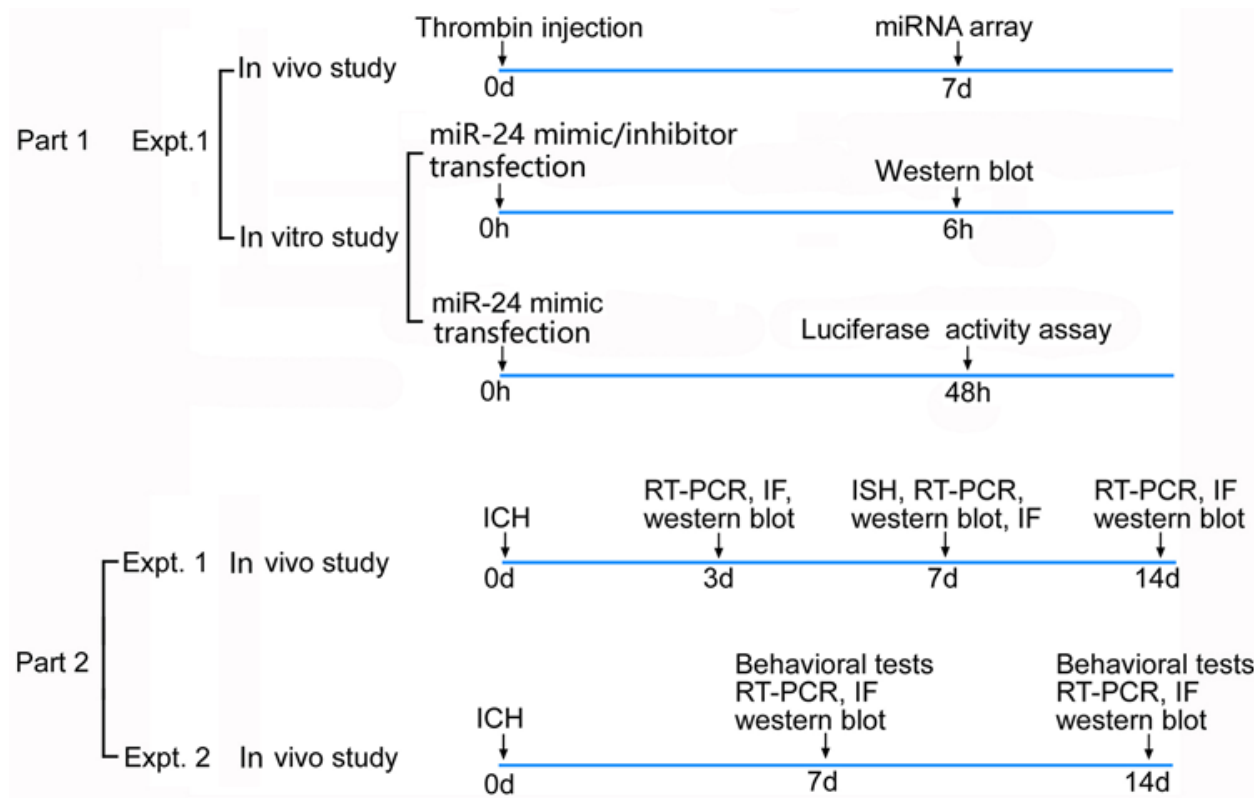

FIG. 1. The timeline of the study design. $d=$ days; Expt. = experiment; $h=$ hours; IF = immunofluorescence. Figure is available in color online only.

preparation (tissues for miRNA array, in situ hybridization, laser capture microdissection, Western blotting, and immunohistochemistry) are also shown in the Supplemental Data.

\section{Lateral Intracerebroventricular Injection of miRNA Antagomir}

The methods for miRNA antagomir preparation and lateral intracerebroventricular injection are described by Ge et al. ${ }^{20}$ miR-24 antagomir or its negative control was diluted to $50 \mu \mathrm{mol} / \mathrm{L}$. Next, 5 - $\mu$ l diluents were added to $12.5 \mu \mathrm{l}$ of lipofectamine-2000 (Life Technologies) and mixed. The mixture was kept at room temperature for 15 minutes. The ICH+antogomir-vehicle animal was injected with $17.5 \mu$ l of lipofectamine-2000. Ten minutes after ICH induction, the mixture was injected at $1.5 \mathrm{~mm}$ posterior to the bregma, $1.1 \mathrm{~mm}$ left of the midline, and $4.5 \mathrm{~mm}$ in depth below the skull over a 10 -minute period. ${ }^{21}$

\section{miRNA Array Assay and Analysis}

The thrombin-infused brains were harvested, and their basal ganglia were separated. Total RNAs were extracted using the RNeasy Mini Kit (Qiagen) according to the manufacturer's procedure. The details for sample RNA quality control are shown in the Supplemental Data. The miRCURY Array Power Labeling kit (Exiqon) was used to label miRNAs. In accordance with miRCURY Array recommending conditions, labeled miRNA samples were hybridized to the MicroRNA microarrays. The Axon GenePix 4000B microarray scanner (Affymetrix Inc.) was used to perform the microarray scan, and the data were extracted from images by GenePix Pro software (version 6.0, Axon Instruments). Data analysis is shown in the Supplemental Data.

\section{Bioinformatics Analysis}

To identify the genes that differentially expressed miRNA targets, the 5 leading miRNA target prediction databases (PicTar, mi-Randa, TargetScan, MicroT, and miRtarget) were used. The enrichment analysis of target genes in biological pathways was performed by using a web-based application, DIANA-mirPath. It was utilized to identify genes in the context of known biological processes, regulatory networks, and other higher-order response pathways.

\section{Luciferase Activity Assay}

The interaction between PHD1 and miR-24 was identified by using the luciferase activity assay in the tool cell line 293T. Details are shown in the Supplemental Data.

\section{miRNA Mimic/Inhibitor Transfection}

The regulatory role of miR-24 on PHD1 was identified by miRNA mimic/inhibitor transfection in the brain microvascular endothelial cell (BMEC) line of rats. miR24 mimics and inhibitors were purchased from RiboBio Co., Ltd. According to the manufacturer's instructions, we used lipofectamine 2000 (Life Technologies) to perform transient transfections. All experiments were performed in triplicate for each group (details shown in the Supplemental Data).

\section{In Situ Hybridization and Immunohistochemistry}

The coexpression of miR-24 and von Willebrand factor (vWF) was detected in near-serial formalin-fixed and paraffin-embedded brain tissue sections $(5 \mu \mathrm{m})$ using in situ hybridization (ISH) and immunohistochemistry (IHC). The brain tissue used for ISH and IHC was located in the right cerebral hemisphere around the hematoma (details are shown in the Supplemental Data). 


\section{Behavioral Tests}

Behavioral tests were performed as previously described $^{5,22}$ and included the body weights, modified Neurological Severity Scores (mNSSs), corner turn test (CTT), and foot-fault test (FFT). The experimental animals were tested on days 3, 7, and 14 after ICH. The details regarding behavioral tests are shown in the Supplemental Data.

\section{Laser Capture Microdissection}

Laser capture microdissection (LCM) combined with immunofluorescence was used to collect endothelial cells (ECs) for subsequent miR-24 isolation and detection (details shown in the Supplemental Data).

\section{Quantitative Real-Time Polymerase Chain Reaction Analysis}

Tissue collected by LCM was used for quantitative real-time polymerase chain reaction (qRT-PCR) analysis. Reverse transcription reactions for miRNA detection $(15 \mu \mathrm{l})$ and TaqMan real-time PCR reactions $(20 \mu \mathrm{l})$ were used. Reverse transcription and TaqMan microRNA assays were performed on a TECHNE TC 512 Instrument and an Applied Biosystems 7000 Instrument, respectively; details are listed in the Supplemental Data.

\section{Western Blot}

The primary antibodies used for the Western blot assay were mouse anti- $\beta$-actin (1:5000, abcam), rabbit antiPHD1 (1:400, Novusbio), and rabbit anti-HIF-1 $\alpha$ (1:500, Santa Cruz Biotech). Enhanced chemiluminescence (Thermo Fisher Scientific) was used for protein visualization. Three independent experiments were performed, and one representative result is shown. The expression level proteins were finally analyzed using Image Lab software densitometrically (details listed in the Supplemental Data).

\section{Immunohistochemistry and Quantification}

Immunohistochemical double-staining was performed to detect proliferating cerebral microvascular ECs on brain sections. The primary and secondary antibodies for neo-nuclei labeling were the mouse anti-proliferating cell nuclear antigen (PCNA; 1:1000, Santa Cruz Biotech) and the anti-mouse Cy3 IgG (1:1000, Jackson ImmunoResearch Laboratories Inc.). The primary and secondary antibodies for cerebral EC detection were the rabbit anti-vWF (1:400, Santa Cruz Biotech) and the anti-rabbit AF488 IgG (1:1000, Jackson ImmunoResearch Laboratories Inc.). The number of $\mathrm{PCNA}^{+} / \mathrm{vWF}^{+}$nuclei around the lesion area was counted using ImageJ analysis software. Details are listed in the Supplemental Data.

\section{Statistical Analysis}

IBM SPSS software (version 23.0, IBM Corp.) was used to perform statistical analysis. The data are presented as the mean \pm SD. Multiple-sample analysis was performed by using 1-way ANOVA followed by the Student-Newman-Keuls test. Differences were considered significant at $\mathrm{p}<0.05$.

\section{Results}

\section{miRNA Expression Profile in Thrombin-Treated Basal Ganglion and Identification of PHD1 as a Target of Candidate miRNAs}

To investigate the miRNAs involved in thrombininduced angiogenesis, we used an miRNA microarray. Five miRNA probes were found to be upregulated by more than 1.5-fold at 7 days after thrombin intervention compared to the opposite hemisphere; these probes were miR-425, miR-872, miR-24, miR-542-5p, and miR-let-7a. miR-499 was downregulated by more than 1.5 -fold after thrombin stimuli (Fig. 2A, Table 1). To find out the molecular target of these differentially expressed miRNAs, we predicted target candidates using the 5 leading algorithmic programs. PHD1 was identified as a candidate target of miR-24 and miR-let-7a. After a literature review, let-7a was excluded, because it was reported to function against angiogenesis.

PHD1 (gene name EGLN2) was among the proangiogenic-related genes that can be regulated by miR-24 (Supplemental Table 2). Computational prediction indicated that miR-24 shares a complementary sequence with the $3^{\prime}$ untranslated region (UTR) of EGLN2. The interaction between EGLN2 and miR-24 was identified by dual-luciferase activity assay. The miR-24 mimic significantly decreased luciferase activity in $293 \mathrm{~T}$ cells transfected with a dual luciferase reporter (psiCHECK2-EGLN2-3'-UTR) for 48 hours compared with its control miRs (3 times), while the mutated miR-24 matching sequence failed to reduce luciferase activity (Fig. 2B).

In order to identify the regulation of miR-24 on its target gene PHDl, the transfection of miR-24 mimic/inhibitor was performed in BMECs of rats. At 6 hours after transfection, Western blotting showed that PHD1 protein in BMECs was decreased by miR-24 mimic transfection and increased by miR-24 inhibitor compared with the negative control ( $\mathrm{p}<0.05$, Fig. $2 \mathrm{C})$. Collectively, the data confirmed the interaction between PHD1 and miR-24.

\section{miR-24 Expressed in ECs After ICH}

ISH combined with IHC was performed at day 7 to show that miR-24 was expressed in ECs in the ICH-affected hemisphere (Fig. 3A).

\section{Hirudin Downregulation of miR-24 in ECs and Hindered Angiogenesis After ICH}

To examine the effects of thrombin on miR-24 in ECs and the further effect on angiogenesis after $\mathrm{ICH}$, hirudin (a thrombin inhibitor) was utilized in this study. vWFpositive ECs were obtained from brain sections by LCM. qRT-PCR showed that the relative miR-24 expression levels peaked on day 7 . The administration of hirudin significantly decreased the levels ( $\mathrm{p}<0.05$; Fig. 3B).

The Western blot assay indicated that the protein level of PHD1 experienced a slump on day 7. ICH downregulated PHD1 $(\mathrm{p}<0.05)$, and this effect was reversed by hirudin $(\mathrm{p}<0.05)$. The expression of HIF-1 $\alpha$ protein showed an opposite trend on days 3 to 14 (Fig. 3C).

Double-labeling immunofluorescence analysis showed that there were few $\mathrm{PCNA}^{+}$nuclei in the sham group. The 

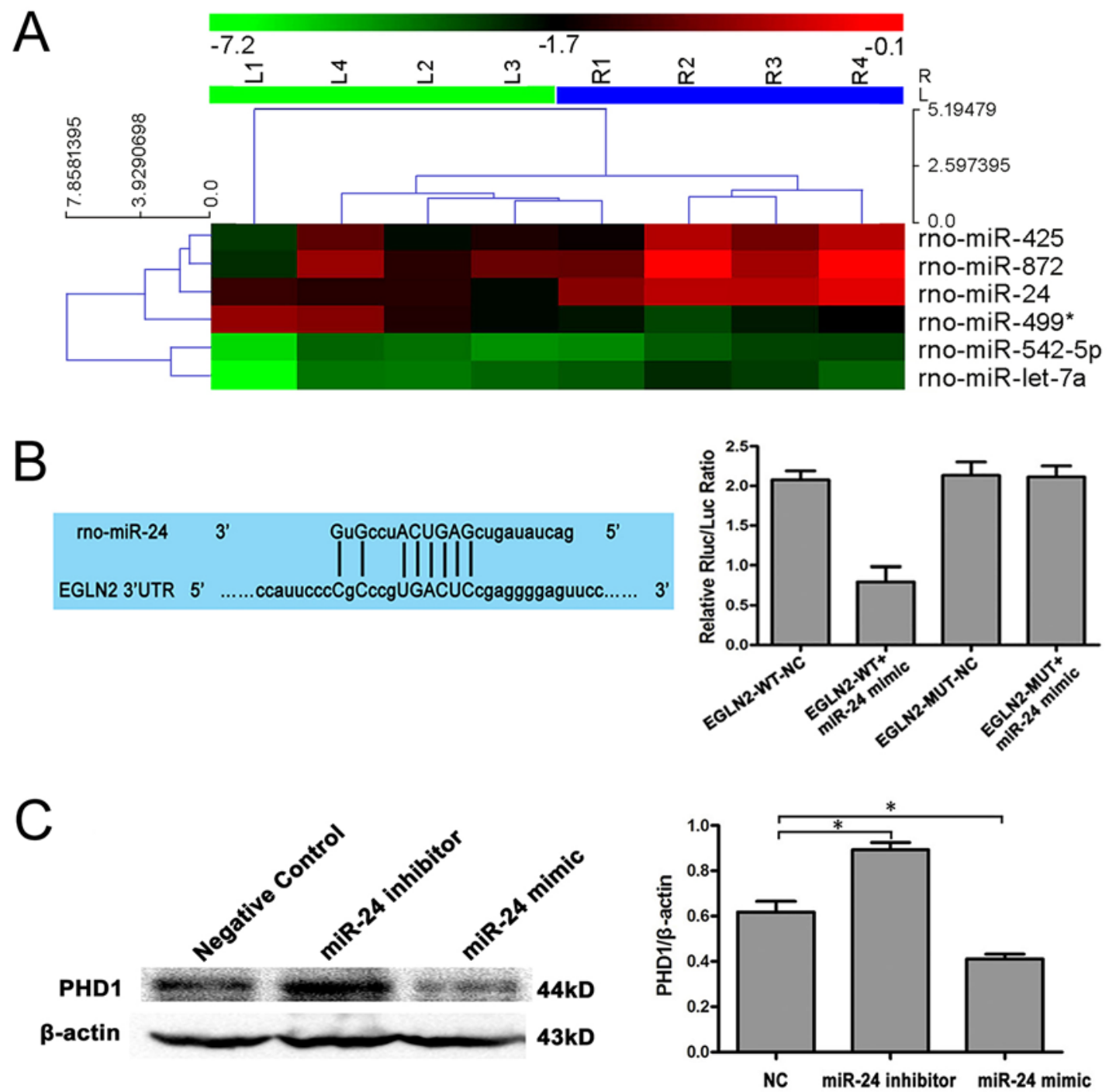

FIG. 2. A: The heat map of differentially expressed miRNAs. The color scale above represents the relative expression levels of miRNA: red represents high expression, green represents low expression $(n=4)$. B: The effect of miR-24 mimic on luciferase activity in psiCHECK2-EGLN2-3'-UTR. The interaction sites between miR-24 and 3'UTR of EGLN2 (left). miR-24 mimic significantly decreased the luciferase activity in 293T cells transfected with a dual luciferase reporter (psiCHECK2-EGLN2-3'-UTR) for 48 hours compared with its control miR [3-fold decrease]; right). MUT = mutated type; $\mathrm{NC}=$ negative control; WT = wild type. C: The effects of miR-24 on PHD1 protein level in BMECs. After transfection for 6 hours, the miR-24 mimic significantly decreased the protein level of PHD1, while the miR-24 inhibitor significantly increased this level ( $\left.{ }^{*} p<0.05\right)$. All experiments were performed in triplicate for each group. Figure is available in color online only.

number of $\mathrm{PCNA}^{+}$nuclei in other experimental groups went up consistently from days 3 to 14 . ICH resulted in far more $\mathrm{PCNA}^{+}$nuclei in microvessels compared with the ICH+hirudin group (Fig. 4).

\section{Inhibition of miR-24 Impeded Neurological Recovery After ICH}

AntagomiR-24 (miR-24 inhibitor) was infused into the right lateral ventricle to confirm the effect of miR-24 on brain recovery after ICH. The sham, ICH+antagomir-control (ICH+antagomir-Co), and $\mathrm{ICH}+$ vehicle groups were set as controls. The sham group showed steady growth of body weight and no neurological impairments. The body
TABLE 1. Statistical results of the set of miRNAs upregulated or downregulated in thrombin-treated tissue compared with contralateral normal tissue

\begin{tabular}{lcccr}
\hline \multicolumn{1}{c}{ miRNA } & $\begin{array}{c}\text { Fold Change } \\
\text { (rt vs It) }\end{array}$ & $\begin{array}{l}\mathrm{p} \mathrm{Value} \\
\text { (rt vs It) }\end{array}$ & $\begin{array}{c}\mathrm{Lt} \\
\text { (mean) }\end{array}$ & $\begin{array}{c}\mathrm{Rt} \\
\text { (mean) }\end{array}$ \\
\hline rno-miR-542-5p & 1.856644192 & 0.04109 & 59.875 & 72.00 \\
\hline rno-miR-425 & 1.698129961 & 0.024196 & 133.13 & 200.50 \\
\hline rno-miR-872 & 1.824440441 & 0.028387 & 159.88 & 263.50 \\
\hline rno-miR-24-1-5p & 1.830421184 & 0.013975 & 144.25 & 239.37 \\
\hline rno-let-7a & 2.331055681 & 0.038958 & 60.00 & 78.13 \\
\hline rno-miR-499 & 0.518922488 & 0.033774 & 168.88 & 110.88 \\
\hline
\end{tabular}


Cui et al.
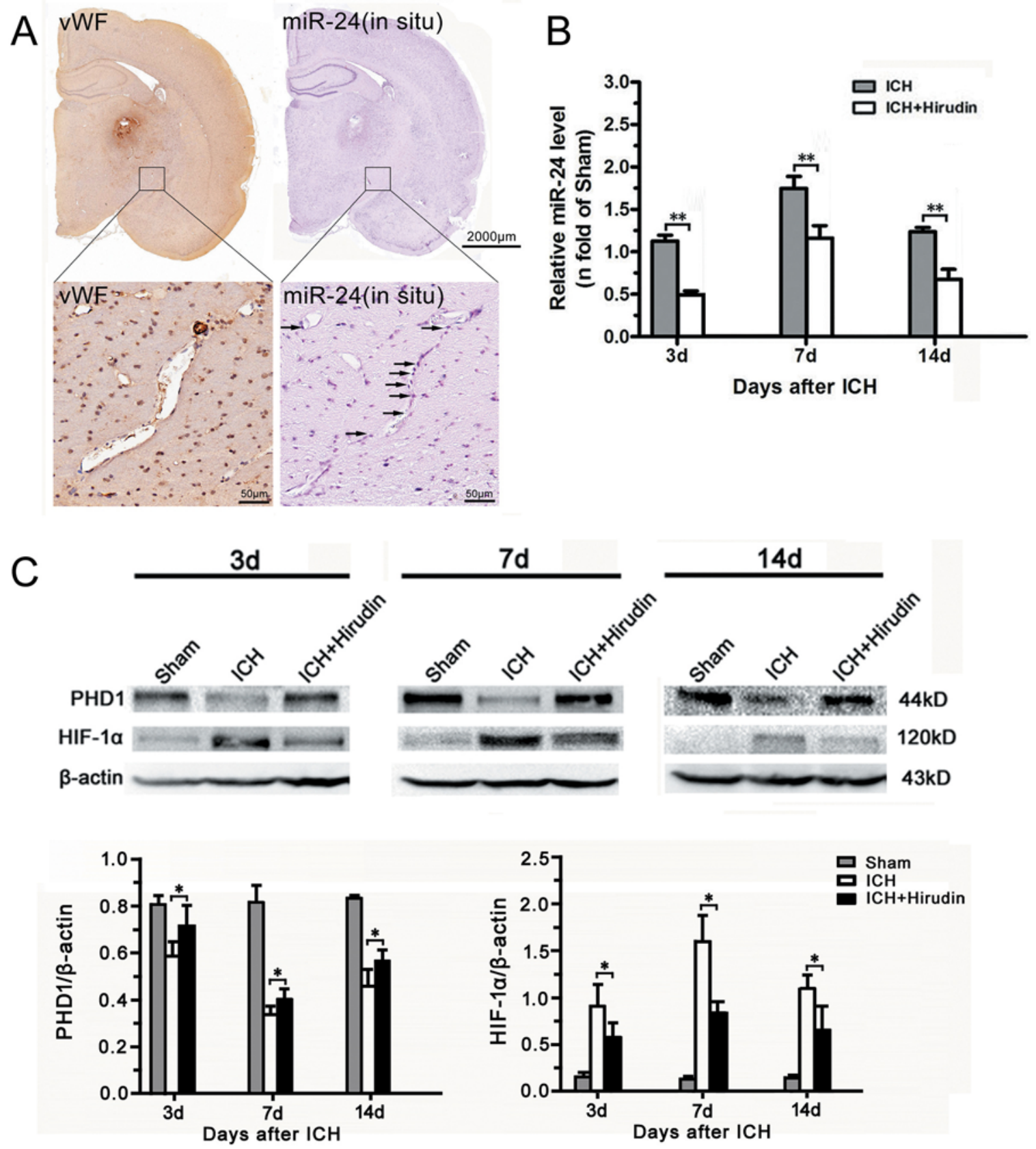

FIG. 3. A: ISH and IHC showed miR-24 expression in ECs in two near-serial hemorrhagic brain sections (upper, 7 days after ICH). The insets represent a higher magnification. ISH showed miR-24 expression (lower right, purple, arrows) was at the same sites with vWF+ ECs (lower left, brown). B: Bar graph of the expression of miR-24: inhibition of thrombin downregulated the expression of miR-24. The level of miR-24 increased 3 days after $\mathrm{ICH}$, peaked at day 7, then decreased. Intervention with thrombin inhibitor hirudin significantly downregulated the level of miR-24 (data represent the mean $\pm S D, n=5$ for each time point in each group; ${ }^{* *} \mathrm{p}<0.01$ ). C: Western blot of PHD1 and HIF-1a: the effect of thrombin inhibitor on the protein levels of PHD1 and HIF-1a. The expression of PHD1 started to decrease from day 3, reached its lowest level at day 7, then showed a moderate increase. Hirudin increased expression of PHD1 at each time point. The expression of HIF-1a protein showed an opposite trend through days 3 to 14 (data represent the mean $\pm S D, n=5$ for each time point in each group; ${ }^{*} p<0.05$ ). Figure is available in color online only.

weight in the antagomiR-24 groups was noticeably lower than that in the ICH groups $(\mathrm{p}<0.05)$. Compared with ICH groups, antagomiR-24 infusion showed markedly higher neurological scores and poorer CTT and FFT performance ( $\mathrm{p}<0.05$; Fig. 5A-D).

\section{Effect of miR-24 on Angiogenesis After ICH}

After antagomir infusion, vWF-positive ECs were obtained from ICH animal brain sections by LCM. qRT-PCR results indicated that antagomiR-24 infusion downregulated the expression of miR-24 at days 7 and 14 after ICH (p 

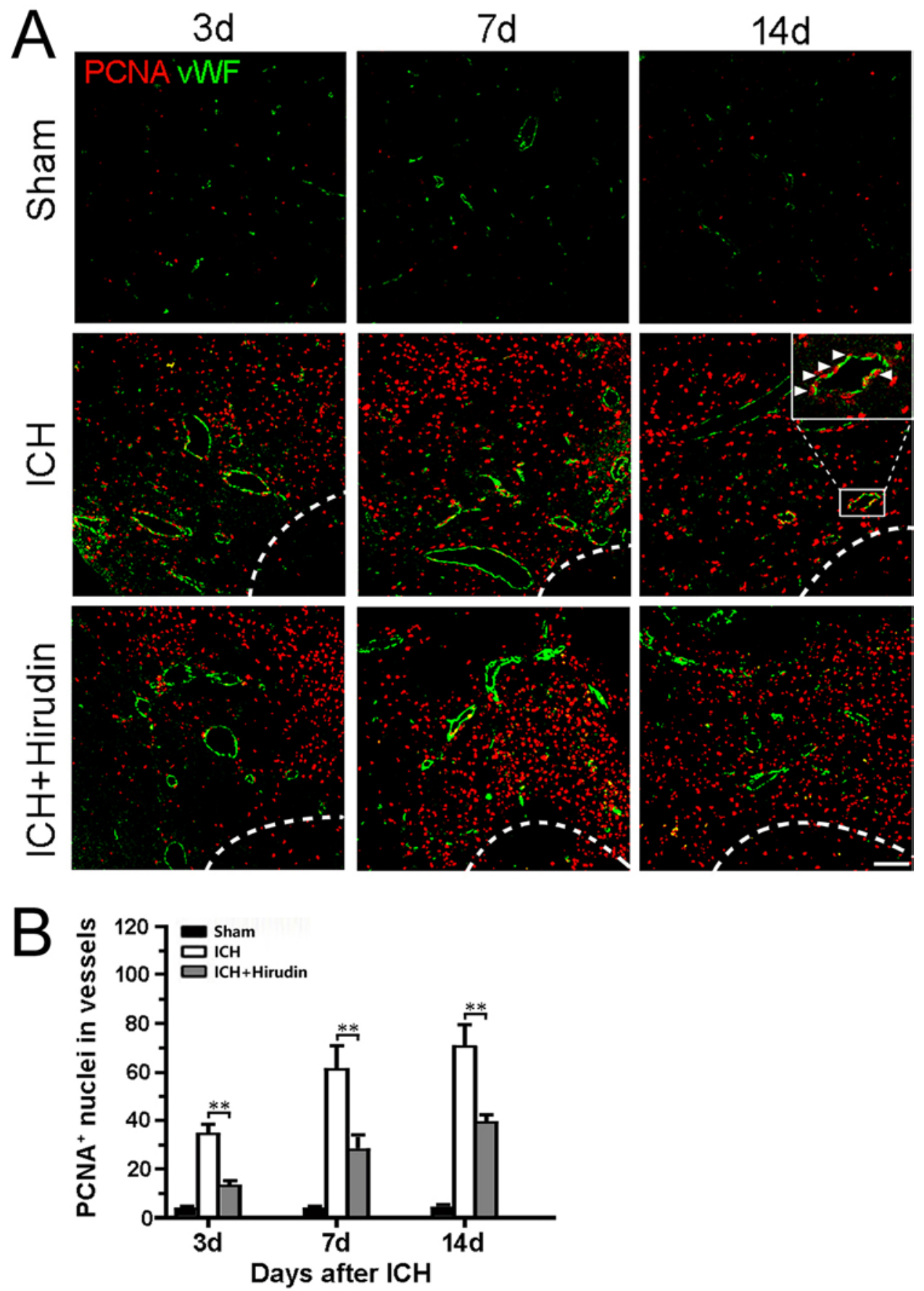

FIG. 4. Immunofluorescence of PCNA+ nuclei in microvessels: the effect of thrombin inhibitor on angiogenesis PCNA ${ }^{+}$nuclei (red) located in $\mathrm{VWF}^{+}$microvessels (green; $\mathbf{A}$ ). There were few $\mathrm{PCNA}^{+} / \mathrm{VWF}^{+}$nuclei in the sham-operated group. Three days after $\mathrm{ICH}$, the number of $\mathrm{PCNA}^{+} / \mathrm{VWF}^{+}$nuclei gradually increased. Hirudin significantly reduced the number of $\mathrm{PCNA}^{+} / \mathrm{vWF}^{+}$nuclei in each time point (B). The inset in ICH 14d is a representative image of a PCNA-positive microvessel (arrowheads) at a higher magnification $(\times 2.5)$. Data represent the mean $\pm S D, n=5$ for each time point in each group; ${ }^{* *} p<0.01$. The dashed line represents the border of the hematoma. Bar $=75 \mu \mathrm{m}$. Figure is available in color online only.

$<0.05$; Fig. 5E). Moreover, antagomiR-24 infusion greatly increased the protein level of PHD1 ( $p<0.05$; Fig. 5F and $\mathrm{G})$ and the number of $\mathrm{PCNA}^{+}$nuclei in $\mathrm{vWF}^{+}$microvessels $(\mathrm{p}<0.05$; Fig. 6).

\section{Discussion}

We demonstrated for the first time that: 1) thrombin upregulated miR-24, which targeted PHD1; 2) ICH enhanced miR-24 expression and angiogenesis, which was 

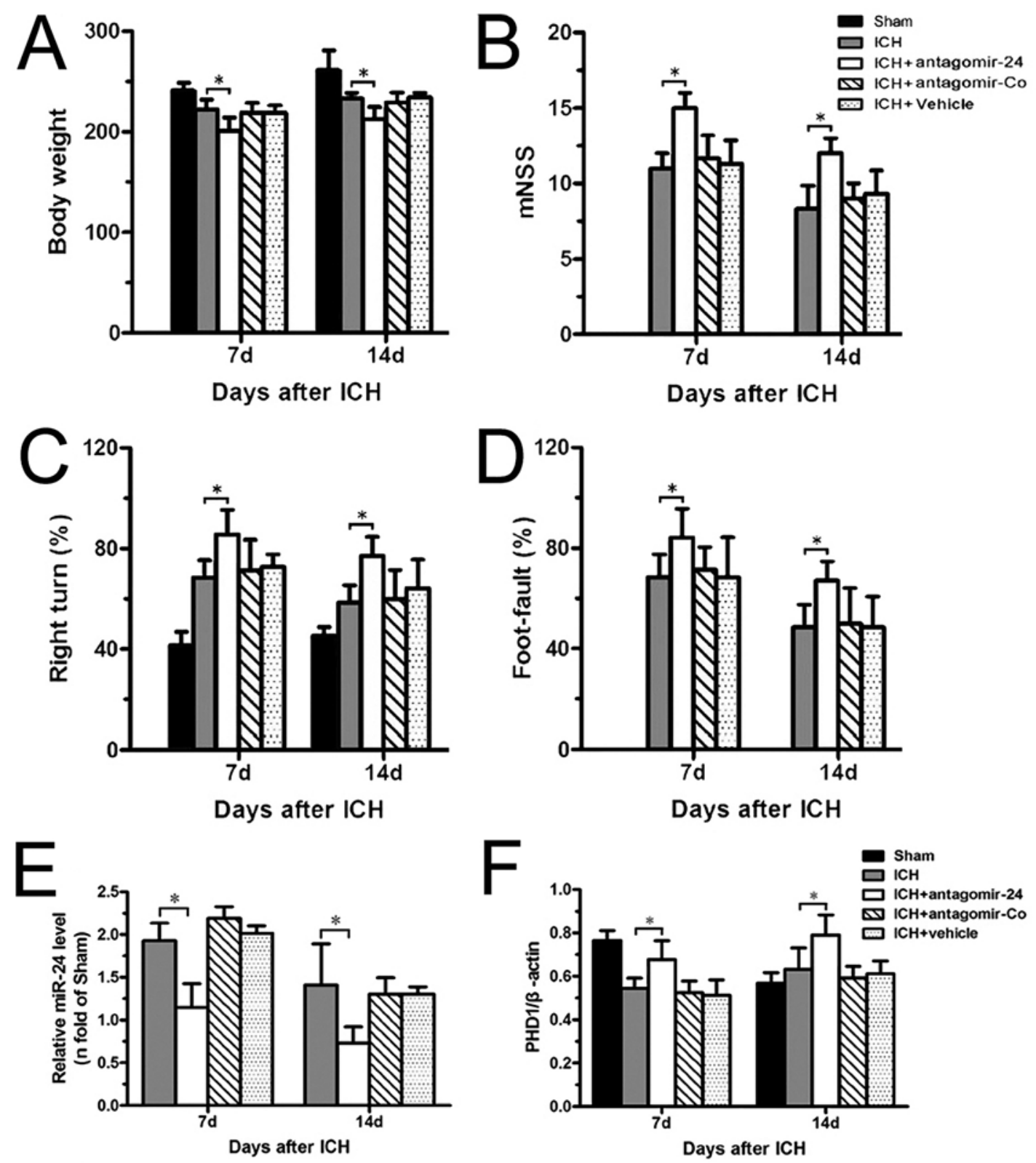

Days after ICH
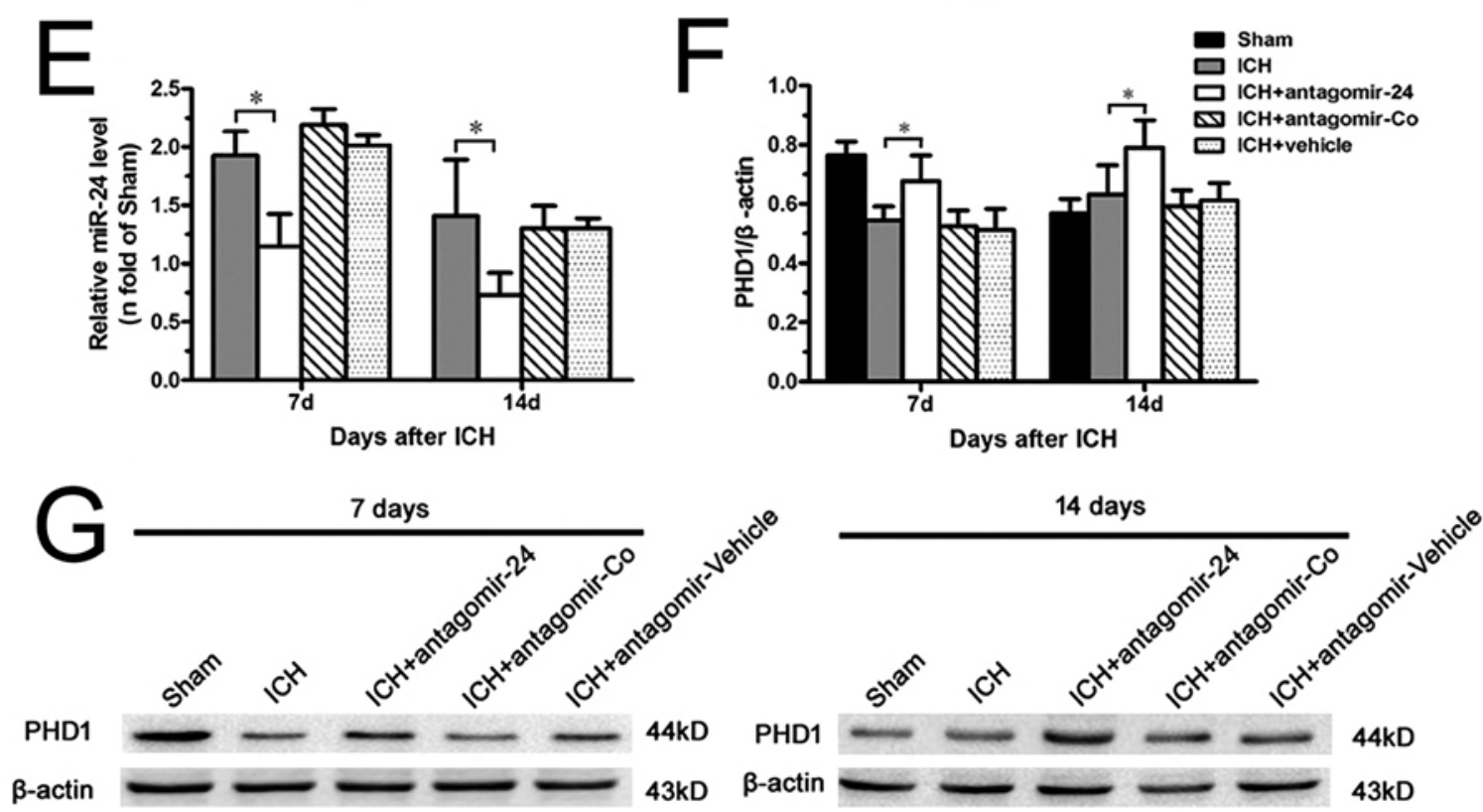

FIG. 5. Bar charts of the results of neurobehavioral tests. A-D: The effect of miR-24 on neurological recovery after ICH. The animals in the antagomiR-24 group had a markedly lower body weight, higher mNSSs, and poorer performance on the CTT and FFT compared with animals in the ICH groups. "antagomir-Co" represents the negative control of antagomiR-24; "vehicle" is the solvent of antagomiR-24. Data represent the mean $\pm S D, n=15$ for each time point in each group; ${ }^{*}<<0.05$. E-G: The effect of miR-24 on the protein level of PHD1. The bar graph of the expression level of miR-24 in the antagomiR-24 group shows that it was significantly lower than that in the ICH group at days 7 and 14 after ICH (E). The Western blot of PHD1 shows that, compared with the ICH group, PHD1 protein was increased in the antagomiR-24 group ( $F$ and $G$ ). Data represent the mean $\pm S D, n=5$ for each time point in each group for qRT-PCR and Western blot analysis, respectively; ${ }^{*} p<0.05$. 


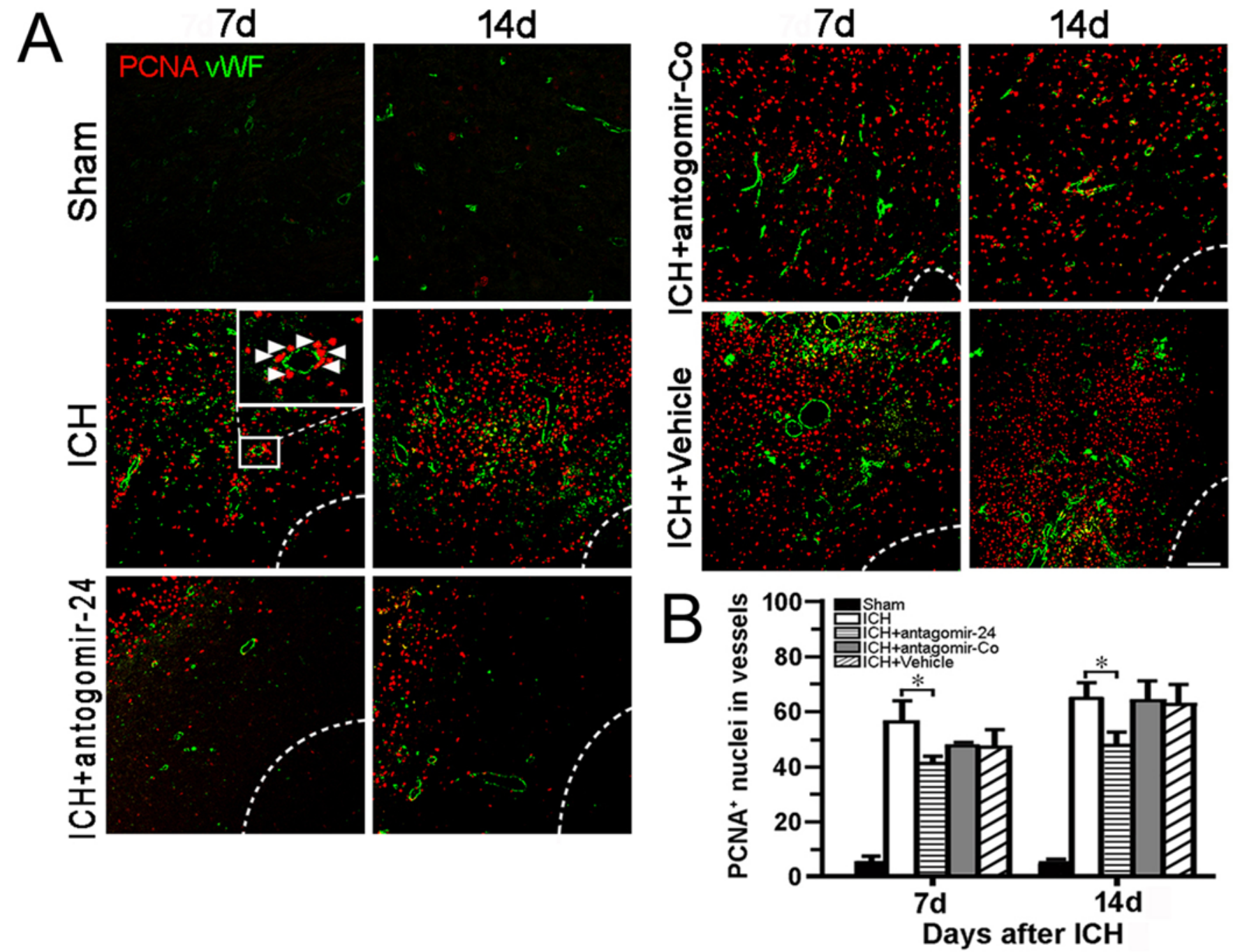

FIG. 6. Immunofluorescence of PCNA+ nuclei in microvessels: the effect of miR-24 on angiogenesis after ICH. PCNA+/vWF ${ }^{+}$nuclei $^{+}$ were decreased in the antagomiR-24 group ( $\mathrm{PCNA}^{+}$nuclei [red] located in vWF+ microvessels [green]). Data represent the mean $\pm S D, n=5$ for each time point in each group; ${ }^{*} p<0.05$. Bar $=75 \mu \mathrm{m}$. The inset in $I C H ~ 7 d$ is a representative image of a PCNA ${ }^{+}$ microvessel (arrowheads) at a higher magnification (x2.5). "antagomir-Co" represents the negative control of antagomiR-24; "vehicle" is the solvent of antagomiR-24. Figure is available in color online only.

reversed by hirudin, a thrombin inhibitor; and 3) miR24 promoted angiogenesis and neurological recovery by depressing PHD1 after ICH. Our findings suggest that thrombin upregulated PHD1-targeting miR-24 to promote angiogenesis after ICH (Fig. 7). These findings revealed a new mechanism for how thrombin, a unique post-ICH angiogenic promotor, initiates the process of angiogenesis after ICH. The findings will help us to improve the understanding of cerebral recovery after hemorrhagic injury and develop novel therapeutic strategies.

\section{Thrombin Elevated miR-24 Expression and Angiogenesis After ICH}

The microarray analysis in the present study showed that miR-24 was significantly upregulated in the thrombininfused basal ganglia compared with those in the contra- lateral one. A clinical study also reported that circulating miR-24 was elevated by approximately 5-fold in ICH patients compared with miR-24 in healthy controls. ${ }^{23}$ However, another study showed an increase in miR-298 and miR-245 in the hippocampus in the blood-infused model and elevation of miR-107, miR-200b, and miR-331-5p in the hippocampus in the thrombin-infused model. ${ }^{24}$ Considering that the basal ganglia are the most $\mathrm{ICH}$-frequent region clinically, we collected this area for miRNA extraction. The expression profile of miRNA is strictly characterized by temporal and spatial (tissue and organ) differences, which might be a major reason for the different miRNAs found in different studies. ${ }^{25}$

Previous studies found that miR-24 was increased under hypoxic conditions, such as in cardiac ischemia, ischemic stroke, and tumors. ${ }^{26-29}$ However, there is limited evidence regarding the occurrence of hypoxia in the perihematomal 


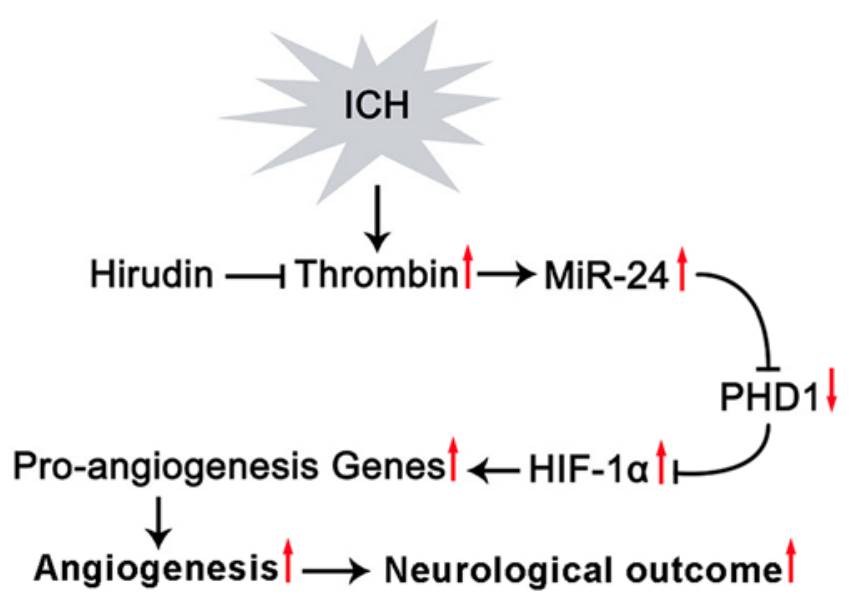

FIG. 7. Diagram showing the effect of thrombin on miR-24 and its target gene PHD1 after ICH. The reduction of HIF-1a degradation and increased angiogenesis after $\mathrm{ICH}$ was triggered by thrombin-induced upregulation of miR-24 via inhibiting PHD1 protein expression. The thrombin inhibitor hirudin can reverse this effect. Figure is available in color online only.

zone. ${ }^{7}$ Thus, hypoxia cannot account for the post-ICH miR24 change. After ICH, abundant thrombin is released from the hematoma, infiltrating into the cerebral parenchyma. ${ }^{22}$ A high dose of thrombin is involved in acute brain injury, causing neuronal death, brain edema, and blood-brain barrier disruption, while a low dose participates in brain recovery, promoting angiogenesis, neurogenesis, and synaptogenesis. ${ }^{3,11,22,30}$ We have demonstrated that it is also a unique signaling molecule for triggering angiogenesis following ICH. ${ }^{3,11}$ In the present study, a substantial increase in miR-24 was detected in thrombin-infused basal ganglia. To verify the role of thrombin in miR-24 expression and downstream responses following $\mathrm{ICH}$, a specific thrombin inhibitor (hirudin) was used. Hirudin deactivates thrombin by simultaneously binding to the active site to form irreversible stoichiometric complexes. ${ }^{31}$ qRT-PCR confirmed that the level of miR-24 increased after ICH in ECs, which were isolated by LCM. EC proliferation was also observed. These phenotypes, however, were repressed by hirudin. Moreover, ISH combined with IHC confirmed miR-24 expression in ECs around the hematoma. It has been demonstrated that miR-24 participates in numerous physiological and pathological processes, including angiogenesis. ${ }^{26,32,33}$ Tube development, endothelial spheroid formation, and cell proliferation were suppressed by miR24 through targeting GATA2 and PAK4 in myocardial infarction, ${ }^{26}$ whereas miR-24 promoted tumor growth and angiogenesis by targeting Bim in pancreatic carcinoma. ${ }^{34}$ Thus, it can be confirmed that thrombin is responsible for miR-24 upregulation, which may be involved in angiogenesis after ICH.

\section{miR-24 Promoted Angiogenesis by Targeting PHD1 After ICH \\ PHD1 Is the Target Gene of miR-24}

We then predicted that PHDI was the candidate target of miR-24 using bioinformatics analysis. The lucifer- ase activity assay further confirmed the complementarity between miR-24 and PHD1 mRNA. The direct interaction proved that BMECs transfected with miR-24 mimic suppressed PHDI expression, which was enhanced by miR-24 inhibitor transfection. Therefore, the data support that miR-24 can inhibit PHD1 mRNA translation through binding to the 3'UTR of the mRNA.

\section{The Effect of miR-24 on the Protein Level of PHD1 and HIF-1a and Angiogenesis After ICH}

PHD1 is located on chromosome 19q13.2 with a length of 1981bp. It is a key prolyl hydroxylase of HIF-1 $1 .{ }^{45}$ In the presence of oxygen, PHD1 mediates HIF-1 $\alpha$ hydroxylation at the 402 and 564 proline residues in the oxygendependent degradation domain. The hydroxylation then induces ubiquitin degradation of HIF-1 $\alpha$ via the interplay with tumor suppressor protein von Hippel-Lindau. ${ }^{35}$ While hypoxia inactivates PHD1, hydroxylation and degradation of HIF-1 $\alpha$ are inhibited. HIF- $1 \alpha$ is a nuclear transcriptional factor and hub mediator of angiogenesis. ${ }^{36,37}$ Our in vivo study further confirmed that enhancement of miR-24 and reduction of PHD1 by hirudin could increase HIF-1 $\alpha$ protein and angiogenesis. Our findings are consistent with the previous observation that the protein level of HIF- $1 \alpha$ was elevated after miR-24 overexpression. ${ }^{26}$ Thus, we inferred that miR-24 might promote angiogenesis by targeting PHD1, which plays the role of degrading HIF-1 $\alpha$. The effect of miR-24 on PHD1 and angiogenesis after ICH was further verified by lateral ventricle injection of antagomiR-24, the antagonist of miR-24. miRNA antagomir is a chemically engineered oligonucleotide. It plays a longlasting and specific role in competitively blocking endogenous miRNAs by binding with mRNAs of target genes. In an in vivo "loss-of-function study," antagomir was used to better understand miRNA mechanisms. ${ }^{38}$ Our study showed that infusion of antagomiR-24 notably suppressed miR-24 expression, increased the protein level of PHD1, and reduced the number of newborn ECs after ICH. The function of miR-24 was also confirmed by the fact that overexpressed miR-24 promoted vascular tube formation in human umbilical vein ECs, whereas the tube formation was blocked when miR-24 was knocked down. ${ }^{34}$ Additionally, the miR-24-mediated cell growth rate was elevated by $80 \%$, while miR-24 downregulation reduced the cell growth rate by nearly half compared with the control. ${ }^{34}$ Therefore, it was concluded that miR-24 promotes ICHinduced angiogenesis by targeting PHD1. A mature and stable newborn microvessel system after brain injury is indispensable for efficient perfusion, which promotes oxygen and metabolite exchange and removes necrotic debris. It is critical for brain recovery and functional outcome improvement.

\section{Effect of miR-24 on Neurological Recovery After ICH}

In behavioral tests, body weight reflects the condition of food intake, water drinking, and motor function in recovery. The mNSS is a sensitive and reliable index in assessing motor function, balance, and flexibility of front limbs. ${ }^{39}$ CTT and FFT are objective and sensible tests for evaluating the deficit in sensorimotor function. They are also particularly advantageous in the assessment of mul- 
tiple sensory and striatal dysfunction-associated motor asymmetry. ${ }^{40,41}$ Body weight, mNSS, CTT, and FFT comprehensively show the neurological recovery and the improvement of quality of life. After ICH, the body weight of rats gradually increased with a better performance on the mNSS, CTT, and FFT. This effect was reversed by miR-24 suppression. Recent evidence has suggested that angiogenesis alleviates the neurological deficit by increasing blood supply for lesioned regions, elevating oxygen and metabolite exchange, and removing the toxic substance and necrotic tissues to promote neurogenesis, synaptogenesis, and axonal elongation.,42,43 Angiogenesis-promoting therapies have been demonstrated to reduce neuronal death, alleviate neurological deficits, and improve overall outcome after ICH. ${ }^{19,44}$ Our previous study also found a close relationship between neurological recovery and angiogenesis. Compared with the ICH group, the group treated with vascular endothelial growth factor receptor inhibitor showed a poorer neurological outcome and fewer proliferated ECs. ${ }^{5}$ Therefore, it is suggested that miR-24 can improve the neurological outcome after ICH by promoting angiogenesis.

However, there were some limitations. First, the miR24-involved pathway is only one of the pivotal signaling mechanisms that impacts post-ICH angiogenesis. It is certain that many other unidentified signaling pathways participate in regulating this process, which will be explored in our future studies. Second, thrombin is a dual-function signal molecule: a potent promoter of cerebral recovery at a low dose, and a strong cellular demolisher at a high dose after ICH. In the present study, the neuroprotective effect of thrombin was the main concern, while the deleterious role of a high dose of thrombin at the early stage of ICH was not a focus. In future studies, the dual effect of thrombin at different doses in different $\mathrm{ICH}$ stages should be comprehensively investigated. Finally, the exact pattern of how thrombin acts on miR-24 was not revealed in the present study.

\section{Conclusions}

The present study suggests thrombin upregulates miR24 after ICH, which promotes angiogenesis by targeting PHD1. This study raises a new possibility of modulating miRNAs to improve ICH prognosis.

\section{Acknowledgments}

This study was funded by grants from the National Natural Science Foundation of China (no. 81904078, to Hanjin Cui; no. 81874425, to Tao Tang; no. 81603414, to Ali Yang; and nos. 81673719 and 81973665 , to Yang Wang) and from the Science Foundation for Young Scientists of Xiangya Hospital, Central South University (no. 2016Q09, to Hanjin Cui).

\section{References}

1. Bernardo F, Rebordão L, Machado S, et al. In-hospital and long-term prognosis after spontaneous intracerebral hemorrhage among young adults aged $18-65$ years. J Stroke Cerebrovasc Dis. 2019;28(11):104350.

2. Wilkinson DA, Pandey AS, Thompson BG, et al. Injury mechanisms in acute intracerebral hemorrhage. Neuropharmacology. 2018;134(Pt B):240-248.
3. Zhou HJ, Tang T, Cui HJ, et al. Thrombin-triggered angiogenesis in rat brains following experimental intracerebral hemorrhage. J Neurosurg. 2012;117(5):920-928.

4. Lan X, Han X, Li Q, et al. Modulators of microglial activation and polarization after intracerebral haemorrhage. Nat Rev Neurol. 2017;13(7):420-433.

5. Cui HJ, Yang AL, Zhou HJ, et al. Buyang huanwu decoction promotes angiogenesis via vascular endothelial growth factor receptor-2 activation through the PI3K/Akt pathway in a mouse model of intracerebral hemorrhage. BMC Complement Altern Med. 2015;15:91.

6. Li HT, Zhou HJ, Zhong JH, et al. 2-methoxyestradiol inhibits intracerebral hemorrhage-induced angiogenesis in rats. Turk Neurosurg. 2018;28(2):241-247.

7. Hanjin C, Tao L, Pengfei L, et al. Altered long noncoding RNA and messenger RNA expression in experimental intracerebral hemorrhage - a preliminary study. Cell Physiol Biochem. 2018;45(3):1284-1301.

8. al Taleb Z, Petry A, Chi TF, et al. Differential transcriptional regulation of hypoxia-inducible factor- $1 \alpha$ by arsenite under normoxia and hypoxia: involvement of Nrf2. J Mol Med (Berl). 2016;94(10):1153-1166.

9. Kuschel A, Simon P, Tug S. Functional regulation of HIF-1 $\alpha$ under normoxia-is there more than post-translational regulation? J Cell Physiol. 2012;227(2):514-524.

10. Gao F, Zheng M, Hua Y, et al. Acetazolamide attenuates thrombin-induced hydrocephalus. Acta Neurochir Suppl. 2016;121:373-377.

11. Yang AL, Zhou HJ, Lin Y, et al. Thrombin promotes the expression of thrombospondin-1 and -2 in a rat model of intracerebral hemorrhage. J Neurol Sci. 2012;323(1-2):141-146.

12. Omidkhoda N, Wallace Hayes A, Reiter RJ, Karimi G. The role of microRNAs on endoplasmic reticulum stress in myocardial ischemia and cardiac hypertrophy. Pharmacol Res. 2019;150:104516.

13. Blasiak J, Watala C, Tuuminen R, et al. Expression of VEGFA-regulating miRNAs and mortality in wet AMD. $J$ Cell Mol Med. 2019;23(12):8464-8471.

14. Lanigan SM, O'Connor JJ. Prolyl hydroxylase domain inhibitors: can multiple mechanisms be an opportunity for ischemic stroke? Neuropharmacology. 2019;148:117-130.

15. Schreiber T, Salhöfer L, Quinting T, Fandrey J. Things get broken: the hypoxia-inducible factor prolyl hydroxylases in ischemic heart disease. Basic Res Cardiol. 2019;114(3):16.

16. Cheng T, Wang W, Li Q, et al. Cerebroprotection of flavanol (-)-epicatechin after traumatic brain injury via Nrf2dependent and -independent pathways. Free Radic Biol Med. 2016;92:15-28.

17. Kilkenny C, Browne WJ, Cuthill IC, et al. Improving bioscience research reporting: the ARRIVE guidelines for reporting animal research. Osteoarthritis Cartilage. 2012;20(4):256-260.

18. Zhou HJ, Yang X, Cui HJ, et al. Leukemia inhibitory factor contributes to reactive astrogliosis via activation of signal transducer and activator of transcription 3 signaling after intracerebral hemorrhage in rats. J Neurotrauma. 2017;34(8):1658-1665.

19. Zhou J, Liu T, Guo H, et al. Lactate potentiates angiogenesis and neurogenesis in experimental intracerebral hemorrhage. Exp Mol Med. 2018;50(7):78.

20. Ge X, Han Z, Chen F, et al. MiR-21 alleviates secondary blood-brain barrier damage after traumatic brain injury in rats. Brain Res. 2015;1603:150-157.

21. Ge XT, Lei P, Wang HC, et al. miR-21 improves the neurological outcome after traumatic brain injury in rats. Sci Rep. 2014;4:6718.

22. Hu E, Hu W, Yang A, et al. Thrombin promotes pericyte coverage by Tie 2 activation in a rat model of intracerebral hemorrhage. Brain Res. 2019;1708:58-68. 
23. Guo D, Liu J, Wang W, et al. Alteration in abundance and compartmentalization of inflammation-related miRNAs in plasma after intracerebral hemorrhage. Stroke. 2013;44(6):1739-1742.

24. Liu DZ, Tian Y, Ander BP, et al. Brain and blood microRNA expression profiling of ischemic stroke, intracerebral hemorrhage, and kainate seizures. J Cereb Blood Flow Metab. 2010;30(1):92-101.

25. Wu P, Zuo X, Ji A. Stroke-induced microRNAs: the potential therapeutic role for stroke. Exp Ther Med. 2012;3(4):571-576.

26. Bang C, Fiedler J, Thum T. Cardiovascular importance of the microRNA-23/27/24 family. Microcirculation. 2012;19(3):208-214.

27. Lin SC, Liu CJ, Lin JA, et al. miR-24 up-regulation in oral carcinoma: positive association from clinical and in vitro analysis. Oral Oncol. 2010;46(3):204-208.

28. Yin JY, Deng ZQ, Liu FQ, et al. Association between mir-24 and mir-378 in formalin-fixed paraffin-embedded tissues of breast cancer. Int J Clin Exp Pathol. 2014;7(7):4261-4267.

29. Zhou J, Zhang J. Identification of miRNA-21 and miRNA-24 in plasma as potential early stage markers of acute cerebral infarction. Mol Med Rep. 2014;10(2):971-976.

30. Yang Y, Zhang M, Kang X, et al. Impaired adult hippocampal neurogenesis and cognitive ability in a mouse model of intrastriatal hemorrhage. Neurosci Lett. 2015;599:133-139.

31. Cheng B, Liu F, Guo Q, et al. Identification and characterization of hirudin-HN, a new thrombin inhibitor, from the salivary glands of Hirudo nipponia. PeerJ. 2019;7:e7716.

32. Liao XJ, Mao WM, Wang Q, et al. MicroRNA-24 inhibits serotonin reuptake transporter expression and aggravates irritable bowel syndrome. Biochem Biophys Res Commun. 2016;469(2):288-293.

33. Lynch SM, McKenna MM, Walsh CP, McKenna DJ. miR-24 regulates $\mathrm{CDKN} 1 \mathrm{~B} / \mathrm{p} 27$ expression in prostate cancer. Prostate. 2016;76(7):637-648.

34. Liu R, Zhang H, Wang X, et al. The miR-24-Bim pathway promotes tumor growth and angiogenesis in pancreatic carcinoma. Oncotarget. 2015;6(41):43831-43842.

35. Hägg M, Wennström S. Activation of hypoxia-induced transcription in normoxia. Exp Cell Res. 2005;306(1):180-191.

36. Bogdanovski DA, DiFazio LT, Bogdanovski AK, et al. Hypoxia-inducible-factor-1 in trauma and critical care. J Crit Care. 2017;42:207-212.

37. Du Y, Ge Y, Xu Z, et al. Hypoxia-inducible factor 1 alpha (HIF-1 $\alpha$ )/vascular endothelial growth factor (VEGF) pathway participates in angiogenesis of myocardial infarction in muscone-treated mice: preliminary study. Med Sci Monit. 2018;24:8870-8877.

38. Yang X, Tang X, Sun P, et al. MicroRNA-15a/16-1 antagomir ameliorates ischemic brain injury in experimental stroke. Stroke. 2017;48(7):1941-1947.

39. Bai R, Gao H, Han Z, et al. Long-term kinetics of immunologic components and neurological deficits in rats following repetitive mild traumatic brain injury. Med Sci Monit. 2017;23:1707-1718

40. Horiquini Barbosa E, Vallim JH, Lachat JJ, de Castro VL. Assessments of motor abnormalities on the grid-walking and foot-fault tests from undernutrition in Wistar rats. J Mot Behav. 2016;48(1):5-12.

41. McBride DW, Wang Y, Adam L, et al. Correlation between subacute sensorimotor deficits and brain edema in rats after surgical brain injury. Acta Neurochir Suppl. 2016;121:317321.
42. Lee HJ, Kim KS, Park IH, Kim SU. Human neural stem cells over-expressing VEGF provide neuroprotection, angiogenesis and functional recovery in mouse stroke model. PLoS One. 2007;2(1):e156.

43. Zhang ZG, Chopp M. Neurorestorative therapies for stroke: underlying mechanisms and translation to the clinic. Lancet Neurol. 2009;8(5):491-500.

44. Pan C, Liu N, Zhang P, et al. EGb761 ameliorates neuronal apoptosis and promotes angiogenesis in experimental intracerebral hemorrhage via RSK1/GSK3 $\beta$ pathway. Mol Neurobiol. 2018;55(2):1556-1567.

45. Chen N, Huang CX, Huang CH, et al. The molecular characterization, expression pattern and alternative initiation of Megalobrama amblycephala Hif prolyl hydroxylase Phd1. Gene. 2018;678:219-225.

\section{Disclosures}

The authors report no conflict of interest concerning the materials or methods used in this study or the findings specified in this paper.

\section{Author Contributions}

Conception and design: Tang, Cui. Acquisition of data: Yang, $\mathrm{H}$ Zhou, Wang, Luo. Analysis and interpretation of data: Tang, Cui, Yang, H Zhou, Wang, Luo, Jun Zhou, Liu, Li. Drafting the article: Cui. Critically revising the article: Cui. Reviewed submitted version of manuscript: Tang. Approved the final version of the manuscript on behalf of all authors: Tang. Statistical analysis: Liu, Li, Jing Zhou. Administrative/technical/material support: Jun Zhou, E Hu, He, W Hu. Study supervision: Tang.

\section{Supplemental Information}

\section{Online-Only Content}

Supplemental material is available with the online version of the article.

Supplemental Data. https://thejns.org/doi/suppl/10.3171/ 2020.2.JNS193069.

\section{Previous Presentations}

The abstract was submitted to the 7th World IntraCranial Hemorrhage Conference (WICH 2019, Granada, Spain, May 19-21, 2019) and BRAIN \& BRAIN PET 2019, the 29th International Symposium on Cerebral Blood Flow, Metabolism, and Function in conjunction with the 14th International Conference on Quantification of Brain Function with PET (Yokohama, Japan, July 4-7, 2019). The abstract was included in poster reviewing sessions of the Journal of Cerebral Blood Flow \& Metabolism by the committee.

\section{Correspondence}

Tao Tang: Institute of Integrative Medicine, Xiangya Hospital, Central South University, Changsha, Hunan, China. tangtaotay@ csu.edu.cn. 